\title{
Suerte, disuasión y subjetivismo penal
}

Luck, deterrence and criminal subjectivism

Sorte, dissuasão, subjetivismo criminal

Chance, dissuasion et subjectivisme pénal

\section{Ramiro Manuel Fihman | Universidad de Buenos Aires}

Revista Derechos en Acción

Año 3/No 8 Invierno 2018, 152-166

DOI: https://doi.org/10.24215/25251678e176

ORCID: https://orcid.org/0000-0001-6999-6473

Recibido: 21/08/2018

Aprobado: 01/09/2018

Resumen: el objetivo del presente trabajo es analizar la cuestión de la influencia de la suerte en el resultado desde una perspectiva de filosofía del castigo penal. Generalmente, la discusión entre los subjetivistas y los objetivistas gira en torno a debates dogmáticos de teoría del delito sin que se advierta otra posible forma de entender el debate, que está relacionada con qué fin consideramos que tiene la pena. Este ensayo parte de la idea de que la importancia que le daremos a la suerte depende de qué teoría del castigo se adopte. Por otro lado, se sostiene que la teoría de la disuasión-o prevención general negativa- tiende necesariamente, desde un punto de vista teórico, al subjetivismo.

Palabras clave: subjetivismo-objetivismo-disuasión-suerte-resultado

Abstract: The objective of this paper is to analyze the question of the influence of luck on the result from a perspective of the philosophy of criminal punishment. Generally, the discussion between the subjectivists and the objectivists revolves around dogmatic debates on the theory of crime without another possible way of understanding the debate, which is related to what purpose we consider to be worthwhile. This essay is based on the idea that the importance we give to luck depends on which theory of punishment is adopted. On the other hand, it is argued that the theory of deterrence - or negative general prevention - necessarily tends, from a theoretical point of view, to subjectivism. 
Keyswords: Subjectivism - Objectivism - deterrence - lucky - result

Resumo: 0 objetivo do presente trabalho é analisar a questão da influência da sorte no resultado desde uma perspectiva de punição criminal. Geralmente a discussão entre os subjetivistas e os objetivisas gira em torno de debates dogmaticos sobre a teoria do delito sem que se indique outra forma possivel de entender 0 debate, que está relacionado a qual propósito consideramos que tem a penalidade. Este ensaio parte da idéia de que a importância que daremos a sorte depende de que teoria do castigo se adote. Por outro lado, se mantêm que a teoria da dissuasão -ou prevenção geral negativa- tende necessariamente, desde um ponto de vista teórico, ao subjetivismo.

Palavras-chave: subjetivismo - objetivismo - dissuasão - sorte - resultado

Résumé: L'objectif de ce présent article est d'analyser la question de l'influence de la chance sur le résultat dans une perspective de la philosophie de la sanction pénale. Généralement, la discussion entre les subjectivistes et les objectivistes tourne autour de débats dogmatiques sur la théorie du délit sans que soit considéré une autre forme possible d'envisager le débat, qui est lié à la question de la finalité de la peine. Cet essai part de l'idée que l'importance que nous accordons à la chance dépend de la théorie de la peine adoptée. D'un autre côté, on avance que la théorie de la dissuasion - ou prévention générale négative - tend nécessairement, d'un point de vue théorique, au subjectivisme.

Mots-clés: subjectivisme - objectivisme - dissuasion - chance - résultat

\section{Introducción}

Las discusiones en torno a cómo debemos juzgar la influencia de la suerte en nuestras acciones está atravesada por muchos conceptos de dogmática penal que los teóricos han desarrollado a lo largo del tiempo. Por lo general se discuten presupuestos de teoría del delito y problemas de casos hipotéticos sumamente improbables para defender una visión de la responsabilidad frente a intentos influidos por la suerte. El subjetivismo y el objetivismo enfrentan, seguramente, en el futuro un largo y sinuoso camino de discusión en el que intentarán 
mostrarnos situaciones hipotéticas en las que una u otra teoría queda sin respuestas.

El subjetivismo nos plantea que lo relevante a la hora de responsabilizar penalmente a un sujeto por una conducta es estrictamente lo que éste tuvo intención de hacer. En otras palabras, no tiene importancia que el resultado se haya concretado o no, siempre y cuando esta variante haya estado determinada por circunstancias que están fuera del control del agente. Dentro de las muchas variantes que existen dentro de esta corriente, todas coincidirían en que no debe establecerse una distinción en la respuesta penal entre quien intentó hacer algo y no lo logró por "mala suerte" y quien sí obtuvo el resultado buscado.

En contraposición, el objetivismo le asigna mucha importancia a los resultados. Plantea, a la inversa, que debemos diferenciar entre el que logró el resultado y el que no lo hizo. Básicamente, el patrón común entre los objetivistas es que hay que castigar menos a quien intentó dañar a otro y no pudo que a quien efectivamente causó un daño.

El objetivo de este trabajo es aportar una perspectiva distinta a la discusión presentada, desde un punto de vista filosófico. En primer lugar, se pretende defender la idea de que la posición que uno adopte (objetiva-subjetiva) depende de la teoría del castigo que esté dispuesto a aceptar. Por otro lado, se realizará un análisis de la teoría de la disuasión -o prevención general negativa- para determinar qué efectos tendría ésta en la relevancia que le damos a la suerte en el resultado.

La hipótesis de este ensayo consiste en que la teoría de la prevención general negativa, teniendo en cuenta sus fines, lleva necesariamente a adoptar la tesis subjetivista, es decir a castigar igualmente los intentos que los delitos consumados.

Para eso, el trabajo se estructurará de la siguiente manera: se comenzará explicando la idea que relaciona la fundamentación del castigo con la postura sobre la relevancia de la suerte en el resultado; luego, se llevará a cabo un análisis de la teoría de la prevención general y sus principales características y 
problemas; en tercer lugar se plantearán los argumentos para sostener la relación de necesariedad existente entre la disuasión y el subjetivismo; por último, se elaborarán conclusiones.

\section{La importancia de la fundamentación del castigo en nuestras consideraciones acerca del valor de la suerte en el resultado}

Como se adelantó en la introducción, existen numerosas discusiones dogmáticas de los expertos en torno a qué razones hay para considerar relevante la influencia de la suerte en el resultado. Sin embargo, es importante tener en cuenta que hay otras cuestiones que pueden tener que ver con la eterna disputa entre estas dos corrientes del derecho penal e, incluso, constituir su esencia. Estoy hablando de las teorías de la fundamentación castigo. Uno de los presupuestos principales de este trabajo es aceptar la idea de que la forma en la pensamos la función que tiene el castigo penal determina cuál será nuestra opinión respecto de las situaciones en las que la suerte interfiere en nuestras acciones. ${ }^{1}$

Para aceptar esta idea -que en algún punto pretende quitar del terreno de la dogmática la discusión y llevarlo al de la filosofía del castigo- propongo preguntarnos si cambiaría en algún sentido nuestra respuesta frente al castigo equiparado a tentativas y a delitos consumados según la postura que tomemos acerca de para qué sirve castigar. Por ejemplo, supongamos que como comunidad castigamos a una persona con fines resocializadores. ${ }^{2}$ Con ese presupuesto, preguntémonos ahora si tendría sentido establecer una diferencia en la cuantificación de la pena

\footnotetext{
1 Nino, Carlos S., "Subjetivismo y objetivismo en el Derecho Penal”, en Revista Jurídica, Universidad Autónoma de Madrid, Número 1², Madrid, España, 1999, pp. 55-56; FletcheR, George, Rethinking Criminal Law, Nueva York, Oxford University Press, 2000, p. 505.

2 Básicamente, esto consistiría en pensar que el castigo cumple la función de volver a insertar a la persona en la sociedad de la cual dejó de formar parte por haber violado las normas. Así, pareciera que la cárcel cumple una función reeducadora, que tiene por fin rehabilitar al sujeto para reencausarlo según un patrón de vida preestablecido que sería el
} 
entre un sujeto que intentó asesinar a su vecino y no lo logró por circunstancias ajenas a su voluntad y otro que, realizando exactamente la misma conducta, logró el resultado "muerte" exitosamente. La respuesta, intuitivamente, es negativa. Esto es así por razones de sentido común: si el castigo busca adecuar las conductas de las personas a los valores morales que manejamos como comunidad, ¿por qué castigaríamos menos al que tuvo mala suerte? Al fin y al cabo, lo que nos incomodaría como comunidad fue su intención, y eso es lo que debemos tener en cuenta si buscamos "resocializarlo".

Lo mismo ocurre si adoptamos una teoría de "prevención especial negativa", mediante la cual sostenemos que nuestro objetivo es encerrar a aquellos sujetos que son peligrosos para la sociedad, para que podamos vivir en armonía en un mundo de "buenas personas". Si lo que queremos entonces es inocuizar a los sujetos que representan un peligro para los derechos de los demás, entonces llegamos a la misma conclusión: no hay razones para darle el "beneficio" de una reducción de pena al que, por ejemplo, erró el disparo a causa del viento produciendo heridas leves; este sujeto es igualmente peligroso que aquel que tuvo la suerte de apretar el gatillo cuando el viento lo favorecía. Por lo tanto, deberíamos castigarlos de igual manera.

Por el contrario, si tomamos una teoría del castigo basada en el principio de daño ${ }^{3}$ o en la protección de bienes jurídicos, las respuestas comienzan a modificarse. Si vemos en el derecho penal una herramienta para proteger, por ejemplo, la integridad física o la vida de las personas, no suena muy coherente castigar de igual forma a quien daña ese bien jurídico que a quien no lo logra. La teoría del daño, en un sentido similar, implica que “... cada ciudadano tiene plena libertad para perseguir sus deseos y fines mientras no dañe a los demás, esto es, mientras no

\footnotetext{
"normal" (Cfr. Bean, Phillip, Punishment: A Philosophical and Criminological Inquiry, Oxford, Martin Robertson, 1981)

3 Villar, Mario, Suerte penal, Buenos Aires, Ediciones Didot, 2016, pp. 212-245.
} 
menoscabe intereses ajenos". ${ }^{4}$ Con este razonamiento, vemos que tenemos un parámetro marcado al respecto: sin dudas existe una diferencia que debemos tener en cuenta entre aquella persona que lesiona un bien jurídico o daña a otros y quien no lo hace.

No es posible sostener una visión resocializadora del castigo penal y ser objetivista; no es posible pretender la neutralización de los sujetos peligrosos y hacer una diferencia entre quien intenta y quien logra; no es posible partir de una teoría del daño y castigar igualmente a quien daña que a quien no lo hace.

Estas sucintas reflexiones tienen por objetivo defender la idea de que efectivamente existe una relación entre la justificación del castigo que adoptemos y nuestra postura frente a cómo debemos juzgar la influencia de la suerte en el resultado de nuestras conductas. Veremos, en el siguiente apartado, qué ocurre con algunas teorías en particular.

\section{La tesis de Nino}

En línea con lo anterior, existen dos corrientes que presentan especiales problemas a la hora de atribuirles una postura frente a la relevancia de la suerte: la prevención general o disuasión y el retribucionismo.

Carlos Santiago Nino, en su libro "Juicio al mal absoluto" sostiene que adoptar una postura retributiva del castigo lleva necesariamente a un subjetivismo puro. ${ }^{5} \mathrm{Su}$ argumento combina distintos conceptos -por demás complejos- pero fundamentalmente argumenta que el retribucionismo, al estar asociado a la idea de inculpación -que según él sólo puede partir de la intención del autor- nos lleva al perfeccionismo moral y, luego, al autoritarismo. Sostiene que "el subjetivismo del retributivismo

4 Von HiRsch, Andrew, "El concepto de bien jurídico y el 'principio de daño'" en Hefendeht, Roland, Von HIRSCH Andrew y WoHLERS, Wolfgang (Eds.), La teoría del bien jurídico ¿fundamento de legitimación del Derecho penal o juego de abalorios dogmático?, Madrid, Marcial Pons, 2016.

5 Nino, Carlos S., Juicio al mal absoluto, Buenos Aires, Siglo XXI, Edición ampliada, 2015, p. 224 (edición original de 1996). 
deviene en parte de la visión de que el estado mental de un individuo (intenciones, decisiones y elecciones) es lo único que el individuo controla". ${ }^{6}$

Sin embargo, no es mi intención en esta oportunidad defender la idea de que el retribucionismo no está relacionado con una postura subjetivista. Creo que los argumentos planteados por $\mathrm{BEADE}^{7}$ para eso son suficientemente sólidos y no merecen más desarrollo.

En cambio, sí me interesa en esta oportunidad cuestionar la idea defendida por Nino de que la teoría más adecuada es la de la prevención general, y que ésta no tiene problemas que lleven al subjetivismo. ${ }^{8}$ Este autor ataca fuertemente al retribucionismo tildándolo de subjetivismo puro, pero, a mi entender, no consigue demostrar que las teorías de la prevención permitieran defender la postura contraria. Cabe resaltar que el filósofo argentino propone la idea de que las teorías de la prevención son "neutrales" frente a la disputa subjetivaobjetiva. ${ }^{9}$ Aquí ya comienza a resultar extraño cómo es posible compatibilizar la idea de que la posición frente a la suerte depende de la teoría del castigo adoptada y luego que la teoría de la prevención general es neutral. Dicho de otro modo, si pensamos que nuestras ideas acerca de la fundamentación de la pena determinan la relevancia que le daremos a la suerte en el resultado, entonces no podemos sostener que una fundamentación basada en la disuasión no implica una toma de postura en la disputa objetivismo-subjetivismo. Sin embargo, Nino, a pesar de intentar defender la "neutralidad" de la prevención, no se esfuerza demasiado por camuflar su asociación de ésta con el objetivismo.

6 Ibid.

7 Beade, Gustavo A., Suerte moral, castigo y comunidad. Un análisis de la relevancia moral de la suerte en el resultado, Madrid, Marcial Pons, 2017, pp. 98-105.

8 En realidad Nino habla de una "teoría consensual de la pena" que combinaría prevención con el requisito del "consentimiento", pero eso no es relevante para este trabajo.

9 Nino, Carlos S., ob. cit., "Subjetivismo y objetivismo...", p. 75. 
Es interesante para analizar el pensamiento de NiNo el siguiente pasaje de su obra, que reza: "Dado que las inclinaciones, disposiciones, intenciones, deseos y creencias de la gente no pueden dañar directamente los intereses de nadie excepto los propios, ellos no deben figurar o asumir rol alguno en las descripciones de los estados de cosas que la ley debe en definitiva intentar prevenir a través del castigo". ${ }^{10}$

En el citado párrafo se pueden deducir varias cuestiones: En primer lugar, queda en evidencia la postura objetivista de Nino, dado que el razonamiento es que aquello que no daña directamente los intereses de nadie, no debe estar alcanzado por el derecho penal. Por otra parte, se muestra también el fin preventivo que lo otorga el autor al castigo, salvando la curiosa importancia que lo otorga al principio de daño: parece utilizar la prevención como un fin de la pena pero al mismo tiempo como un medio para evitar conductas dañosas. En último lugar, creo que su razonamiento presenta una interesante trampa: si bien es cierto que las inclinaciones, disposiciones, deseos y creencias no pueden dañar intereses ajenos, Nino introduce en esa misma categoría a las intenciones, y he aquí el punto problemático. ¿Qué tan seguros estamos de que las intenciones no pueden dañar a terceros? En principio, pareciera que esto depende de a qué le llamemos "intención": si con este término sólo buscamos hacer referencia lo que queremos hacer -en nuestra mente- sin que exista ninguna manifestación en el mundo real, entonces Nino está en lo cierto. Sin embargo, desde mi punto de vista, lo que Nino está intentando defender es que los intentos de otros no pueden frustrar nuestro plan de vida, y frente a esa afirmación se presentan graves problemas. ¿No interfiere acaso en nuestro plan de vida que alguien intente matarnos? ¿Somos capaces de afirmar que la autonomía de la voluntad de una mujer permanece intacta si intentan violarla? En las siguientes líneas, y tomando este tipo de interrogantes como disparadores, se intentarán demostrar

10 Nino, Carlos S., ob. cit., Juicio al mal..., p. 230. 
las profundas identidades existentes entre la teoría prevencionista y el subjetivismo.

\section{Intentos y resultados. ¿Qué queremos prevenir?}

Volviendo a la teoría de la prevención, lo que ésta plantea es que, si nosotros como comunidad castigamos a una persona por una determinada conducta, se reducirá el número de personas que cometan ese mismo delito. Al parecer -y sin entrar en los debates acerca de la dificultad empírica que esto implicacuando castigamos a alguien se generaría un efecto disuasorio sobre el resto de la comunidad.

Esto necesariamente debe partir del presupuesto liberal de que nos gustaría vivir en un mundo en el que otras personas no interfieran en nuestro plan de vida, por ejemplo, matándonos. Entonces, si deseamos vivir en paz sin que nadie viole nuestra autonomía, entonces parece lógico castigar a quienes lo hagan para que, aquellos que tenían pensado repetir esa conducta, se abstengan de hacerlo.

Creo que el principal problema es el siguiente: lo que buscamos como comunidad no es exactamente prevenir que se cometan delitos, sino que es prevenir que se intente cometer delitos. En otras palabras, la teoría de la prevención, a mi entender, no debería buscar que las personas no consigan asesinar a otras, sino que las personas no pretendan hacerlo. Para demostrar esto propongo el siguiente ejemplo: supongamos que vivimos en una sociedad que castiga con pena de muerte el hurto porque tiene mucho interés en que esto no ocurra. Mi punto es que la esencia de ese interés no aspira a una comunidad en la que los hurtos se ven impedidos por circunstancias ajenas a la voluntad del "delincuente". Si contáramos con los recursos como para tener personal de las fuerzas de seguridad en cada esquina de nuestra ciudad, no estaríamos conformes con la idea de ir caminando por la calle y que cinco personas en unos pocos metros intenten hurtar nuestro teléfono celular pero la policía los capture luego de unos segundos. Lo que 
buscamos en realidad con la amenaza de la pena de muerte es que esas personas se abstengan de intentar hurtar nuestras pertenencias. Me atrevo a afirmar que nadie quiere vivir en un mundo en donde lo único que se evita es el resultado, sino que los intentos de violar nuestro plan de vida, en sí mismos, nos generan malestar.

En ese sentido, creo que lo que busca la justificación preventiva es que en nuestra comunidad las personas no intenten llevar a cabo esas conductas que no nos parecen adecuadas. Por lo tanto, y aquí el punto neurálgico del argumento, sería necesario castigar a aquellas personas que intentan cometer delitos.

Si tuviéramos que diseñar un sistema penal en el que el castigo es disuasivo -y esto estuviera probado- nuestro sentido común nos llevaría a mostrarle a todos que, por ejemplo, "X" persona intentó asesinar al Presidente y, por lo tanto, recibirá un castigo. El mensaje en esa respuesta estatal es "ustedes no deben hacer algo como lo que ' $\mathrm{X}$ ' hizo, porque serán castigados de la misma manera". Pero lo que "X" hizo no fue matar al Presidente, sino intentarlo. Si lo dejáramos libre, siguiendo la lógica prevencionista, probablemente nadie tendría reparos en intentar cometer el mismo delito, y eso no es lo que deseamos: deseamos que todos se abstengan de intentarlo.

Para reforzar aún más esta teoría, podemos tomar el ejemplo que utiliza Nino en Ética y Derechos Humanos, que es la pena de muerte. Allí sostiene que "si se demostrara empíricamente que la ejecución de los que cometen cierto tipo de homicidio previene en forma efectiva un mayor número de muertes que las que resultan de las ejecuciones (y se supone que no hay otros bienes para tomar en cuenta), parece que un criterio meramente cuantitativo de comparación entre vidas humanas conduce a la conclusión de que la sociedad en conjunto resultará beneficiada con la pena de muerte". ${ }^{11}$ Si bien el autor no está de acuerdo con una justificación puramente utilitarista de la pena de muerte, no cuestiona el razonamiento citado, y a mi

11 Nino, Carlos S., Ética y Derechos Humanos, Buenos Aires, Astrea, 1989, p. 452. 
entender hay un detalle importante que debe ser analizado: ¿es efectivamente el número de homicidios cometidos el parámetro que deberíamos tomar para evaluar el funcionamiento de la teoría preventiva? Yo creo que no. Sostengo que, en todo caso, la estadística que deberíamos tener en cuenta si defendiéramos esta postura es el número de intentos de homicidio que se llevaron a cabo desde la vigencia de la pena de muerte, sin importar si fueron consumados o no.

En "Ilícito personal y participación", SANCINETTI presenta el caso de un niño que arroja una piedra al parabrisas del automóvil de su padre, pero, como el golpe se produce justo en un ángulo cromado del mismo, no se daña el vidrio ni se nota siquiera dónde impactó la piedra. Entonces, el padre le dice su hijo que "si hubiera roto el vidrio, tendría prohibido salir a jugar en todo el año". El hermano del niño -muy sensato, por ciertoque fue testigo de la situación, reflexiona internamente acerca de qué quiso el padre al decirle eso a su hermano: “¿que la próxima vez que arroje la piedra vuelva a dar en el ángulo para no romper el cristal o que no vuelva a arrojar una piedra?". ${ }^{12}$ Frente a esto, el autor pretende mostrar una supuesta incoherencia del llamado "disvalor de resultado" con el argumento de que lo que importa no es el daño que causó el niño sino cuál fue su intención. Pero creo que lo interesante de este ejemplo es cómo permite ver el rol de la prevención y su relación con la suerte en el resultado. Supongamos que el padre castiga o reta fuertemente al niño para que éste comprenda que no debe volver a arrojar la piedra. El padre no estaría conforme si su hijo continúa arrojando piedras eternamente pero todas dan en el ángulo del parabrisas, sino que busca que éste no intente dañar el vidrio, justamente porque el resultado estaría librado a la suerte. Esto es lo que hacemos cuando castigamos con fundamentos disuasivos: intentamos reducir los intentos de esa misma conducta. "De la premisa de que la ley debe prevenir perjuicios a terceros, y de que la acción a disuadir debe ser de aquellas

12 SAncinetti, Marcelo A., llícito personal y participación, Buenos Aires, Ad-Hoc, 1997, pp. 19-20. 
que la ley trató de prevenir, no se deriva ninguna conclusión a favor de la relevancia del resultado posterior, todo lo contrario: el modelo de disuasión sólo exige lo siguiente: tú no debes realizar un comportamiento del que se pueda derivar un perjuicio a terceros." 13

Frente a esto, Nino agrega a su defensa de la tesis de la diferencia desde el prevencionismo el argumento de que no es necesario castigar igualmente la tentativa que el delito consumado porque cuando una persona quiere llevar a cabo una conducta antijurídica, se representa la pena que podría imponérsele, pero siempre con los valores cuantitativos de la pena del delito consumado (nadie calcula el proporcional de tentativa a la hora de evaluar cometer o no un delito). En ese mismo sentido, sostiene que la tentativa sí debe ser penada -siempre en menor proporción- porque, de lo contrario, no habría nada que perder para intentar, por ejemplo, robar un banco. ${ }^{14}$ Creo que este punto es parcialmente cierto. La primera idea, de que las personas se representan el castigo del delito consumado parece ser atinada. Sin embargo, la segunda -que busca explicar por qué hay que penar la tentativa- tiene un problema: si sostenemos que nadie tiene en cuenta a la hora de cometer un delito cómo se castiga la tentativa sino únicamente el delito consumado, entonces no podemos sostener que si no se castiga la tentativa todos dejarían de pensar en la pena del delito consumado para tener en cuenta solamente la ausencia de castigo por el intento. Nuevamente, creo que el problema de Nino está en defender la teoría de la prevención. ${ }^{15}$

\footnotetext{
13 Sancinetti, Marcelo A., ob. cit., p. 23.

14 Nino, Carlos S., ob. cit., "Subjetivismo y objetivismo...", p. 79.

15 Algunos podrían sostener que a Carlos Nino le asiste razón por el hecho de que en su teoría de la pena agrega otros elementos además de la prevención, como por ejemplo el llamado "principio de protección prudencial de la sociedad", compuesto por los requisitos de eficacia (que la aplicación tenga éxito a la hora de evitar los males que se pretenden evitar), economía (que no exista una forma de castigar que implique un mal menor), equilibrio (los males que implica la aplicación de la pena deben ser menores que los prevenidos) e inclusión (los males sufridos por aquéllos a los que se impone la pena deben ser tenidos en cuenta a la
} 
Creo que es acertado -y está en sintonía con lo planteado en este trabajo- lo planteado por David Lewis, ${ }^{16}$ que propone un caso en el que dos personas le disparan simultáneamente a un enemigo en común, con las mismas intenciones de asesinarlo, pero uno de ellos yerra el golpe por azar. Frente a esto, el autor nos dice que ambos han participado igualmente de la acción que queremos prevenir con la disuasión. Sostiene que "para prevenir los intentos de éxitos, prevenimos los intentos en general", ${ }^{17} \mathrm{y}$ que no es posible prevenir resultados separadamente de la prevención de los intentos, dado que los que intentan no deciden de forma independiente si desean tener éxito en su conducta.

Esta cuestión, desde mi punto de vista, es central. La imposibilidad de prevenir únicamente intentos exitosos nos lleva a aceptar la idea de que la fundamentación disuasiva del castigo busca prevenir que las personas intenten violar las reglas que tenemos, y no busca prevenir que se produzcan resultados. Por lo tanto, si defendemos la prevención general, no tenemos buenas razones para darle un valor diferenciador a la suerte en el resultado.

\section{Conclusiones}

El objetivo de este trabajo fue aportar una mirada de la discusión sobre el valor de los resultados para la responsabilidad penal desde un punto de vista de la fundamentación del castigo. Para eso, fue preciso demostrar un punto de partida necesario: la teoría del castigo que cada persona adopte, lo llevará a pensar

\footnotetext{
hora de establecer el "equilibrio"). Creo que estos elementos no forman parte de su teoría del castigo. Por el contrario, son condiciones que el autor agrega para pensar en un sistema penal que sea de su agrado, pero nada de ello modifica las razones de fondo por las cuales Nino considera que debemos castigar a otros. En ese sentido, lejos de constituir un argumento para alejar a la teoría de la disuasión del subjetivismo, sólo hace un poco más aceptable su teoría de prevención general en términos prácticos.

16 LewIS, David, "The punishment that leaves something to chance", en Philosophy \& Public Affairs, Vol. 18, No. 1, 1989, pp. 53-67.

17 Traducción propia.
} 
de una forma determinada la responsabilidad penal y, en particular, la disputa entre el objetivismo y el subjetivismo.

Luego se explicó la teoría planteada por Carlos Santiago Nino en relación a su forma de entender el castigo penal, relacionada con la prevención general. Con posterioridad a resumir las críticas del autor al retribucionismo y su supuesta relación con el perfeccionismo moral, se plantearon algunos interrogantes que pretenden poner en duda algunos presupuestos de su defensa de la tesis disuasoria.

En particular, se buscó sostener la idea de que la prevención general lleva necesariamente al subjetivismo. Para eso, se utilizó un argumento de índole filosófico, que puede resumirse muy sucintamente en la siguiente idea: el castigo nunca busca prevenir resultados en sí mismos, sino intentos. Al buscar prevenir intentos -dado que el objetivo de nuestra comunidad es vivir en "paz" sin que otros intenten dañarnos- no hay buenas razones para pensar en beneficiar con menos castigo a quien tuvo suerte y no pudo lograr el resultado. A modo de aclaración, la idea de esta propuesta no es defender la tesis subjetivista, sino que es mostrar que, lejos de ser "neutral", la teoría de la prevención nos haría pensar necesariamente de esta manera y, por lo tanto, desechar la relevancia de la suerte en el resultado.

\section{Bibliografía}

BeAde, Gustavo A., Suerte moral, castigo y comunidad. Un análisis de la relevancia moral de la suerte en el resultado, Madrid, Marcial Pons, 2017.

Bean, Phillip, Punishment: A Philosophical and Criminological Inquiry, Oxford, Martin Robertson, 1981.

FleTCHER, George, Rethinking Criminal Law, Nueva York, Oxford University Press, 2000.

LEwIS, David, "The punishment that leaves something to chance", en Philosophy \& Public Affairs, Vol. 18, No. 1, 1989.

Malamud Goti, Jaime, Suerte, acción y responsabilidad: un ensayo sobre suerte, moralidad y castigo, Bogotá, Editorial Universidad del Rosario, 2008. 
Nino, Carlos S., "Subjetivismo y objetivismo en el Derecho Penal", en Revista Jurídica, Universidad Autónoma de Madrid, Número $1^{\circ}$, Madrid, España, 1999.

Nino, Carlos S., Ética y Derechos Humanos, Buenos Aires, Astrea, 1989.

NIno, Carlos S., Juicio al mal absoluto, Buenos Aires, Siglo XXI, Edición ampliada, 2015.

SANCINETTI, Marcelo A., Ilicito personal y participación, Buenos Aires, Ad-Hoc, 1997.

SAncinetTi, Marcelo A., Teoría del delito y disvalor de acción, Buenos Aires, Hammurabi, 2005.

Villar, Mario, Suerte penal, Buenos Aires, Ediciones Didot, 2016.

Von Hirsch, Andrew, "El concepto de bien jurídico y el 'principio de daño'" en Hefendehl, Roland, Von Hirsch Andrew y Wohlers, Wolfgang (Eds.), La teoría del bien jurídico ¿fundamento de legitimación del Derecho penal o juego de abalorios dogmático?, Madrid, Marcial Pons, 2016. 